\title{
Identifying miRNA-disease association based on integrating miRNA topological similarity and functional similarity
}

\author{
Qingfeng Chen ${ }^{1,2}$, Zhao Zhe ${ }^{1}$, Wei Lan ${ }^{1, *}$, Ruchang Zhang ${ }^{1}$, Zhiqiang Wang ${ }^{2}$, Cheng Luo ${ }^{3}$, \\ Yi-Ping Pheobe Chen ${ }^{4}$ \\ 1 School of Computer, Electronic and Information, Guangxi University, Nanning 530004, China \\ 2 The State Key Laboratory for Conservation and Utilization of Subtropical Agro-bioresources, Guangxi University, Nanning \\ 530004, China \\ 3 Xingjian College of Science and Liberal Arts, Guangxi University, Nanning 530004, China \\ 4 Department of Computer Science and Computer Engineering, La Trobe University, Melbourne, Vic 3086, Australia \\ * Correspondence: lanwei@gxu.edu.cn
}

Received April 28, 2019; Revised June 12, 2019; Accepted June 25, 2019

\begin{abstract}
Background: MicroRNAs (miRNAs) are a significant type of non-coding RNAs, which usually were encoded by endogenous genes with about 22 nt nucleotides. Accumulating biological experiments have shown that miRNAs have close associations with various human diseases. Although traditional experimental methods achieve great successes in miRNA-disease interaction identification, these methods also have some limitations. Therefore, it is necessary to develop computational method to predict miRNA-disease interactions.

Methods: Here, we propose a computational framework (MDVSI) to predict interactions between miRNAs and diseases by integrating miRNA topological similarity and functional similarity. Firstly, the CosRA index is utilized to measure miRNA similarity based on network topological feature. Then, in order to enhance the reliability of miRNA similarity, the functional similarity and CosRA similarity are integrated based on linear weight method. Further, the potential miRNA-disease associations are predicted by using recommendation method. In addition, in order to overcome limitation of recommendation method, for new disease, a new strategy is proposed to predict potential interactions between miRNAs and new disease based on disease functional similarity.

Results: To evaluate the performance of different methods, we conduct ten-fold cross validation and de novo test in experiment and compare MDVSI with two the-state-of-art methods. The experimental result shows that MDVSI achieves an AUC of 0.91 , which is at least 0.012 higher than other compared methods.

Conclusions: In summary, we propose a computational framework (MDSVI) for miRNA-disease interaction prediction. The experiment results demonstrate that it outperforms other the-state-of-the-art methods. Case study shows that it can effectively identify potential miRNA-disease interactions.
\end{abstract}

Keywords: miRNA-disease association; CosRA index; miRNA functional similarity; recommendation method

Author summary: Rapidly increasing evidences show that miRNAs have close relationships with many diseases. Therefore, identifying miRNA-disease interactions may contribute to understand the pathogenesis of diseases. In this paper, we propose a computational framework to predict miRNA-disease interactions based on recommendation method. The experiment results show that our method outperforms other the-state-of-the-art methods.

\section{INTRODUCTION}

MiRNAs are a class of non-coding small RNAs with 20 to
25 nucleotides in length, which play important roles in various biological processes such as cell proliferation, cell differentiation, cell aging, cell development [1]. The 
biogenesis of miRNA is like that: firstly, the primary miRNA (pri-miRNA) is generally transcribed from introns (or host gene) by RNA polymerase II. Then, the pri-miRNA will be processed into the precursor miRNA (pre-miRNA) with about 60 nucleotides in length by Drosha nuclease [2]. Further, the pre-miRNA is transported to the cytoplasm by Exportin-5. Finally, the mature miRNA, which can target the mRNA to regulate the expression of gene, is formed from pre-miRNA with RNase III enzyme. During the past decades, increasing studies have shown that miRNAs have close relationships with various human diseases such as breast cancer [3], liver cancer [4], and gastric carcinoma [5]. Therefore, identifying associations between miRNAs and diseases may contribute to uncover the pathogenesis of human diseases.

Since the first discovery of miRNA (lin-4) about 30 years ago, many miRNAs have been identified based on experimental methods [6]. However, these methods are time consuming and expensive. In order to overcome these limitations, some computational methods have been proposed to predict miRNA-disease associations [7-11]. Jiang et al. [12] proposed a computational model based on hypergeometric distribution to predict miRNA-disease association by integrating the miRNA functional similarity, disease phenotype similarity network and known miRNA-disease associations. Xuan et al. [13] presented a computational method named HDMP, to discover the relationships of miRNAs and diseases based on weighted $k$ most similar neighbors. This method calculated the functional similarity by integrating the similarity of information content of disease terms and phenotype similarity between diseases. Mørk et al. [14] developed an approach called miRPD to predict disease-related miRNAs. Chen et al. [15] introduced regularized least squares to discover the potential relationships between miRNAs and diseases. Peng et al. [16] presented a new method to predict miRNA-disease association by integrating microRNA, disease, gene and environment factor networks. Yan et al. [17] proposed a computational approach based on logistic matrix factorization to identify interactions between miRNAs and diseases. Luo et al. [18] developed a computational approach based on unbalanced birandom walk for predicting microRNA-disease associations. Lan et al. [19] utilized kernelized Bayesian matrix factorization method to infer potential miRNA-disease associations by computing sequence and functional similarity of miRNA, semantic similarity of disease. Liu et al. [20] presented a computational method to predict miRNA-disease interactions based on random walk. Zou et al. [21] proposed two methods, KATZ and CATAPULT, to identify potential miRNA-disease associations based on known similarity network analysis. Although all these existing methods have achieved great successes, the predicting accuracy still needs to be improved.

In this paper, we propose a computational framework to predict associations between miRNAs and diseases based on recommendation method. Firstly, the miRNA-miRNA similarity is calculated based on miRNA-disease topological feature and miRNA functional information. The disease-disease similarity is calculated based on diseasegene association. Then, the miRNA similarities are integrated to improve the reliability of miRNA similarity. Further, the potential miRNA-disease associations are predicted by using recommendation method. In addition, to over the limitation of existing recommendation method, a method is proposed for identifying interactions between miRNAs and new disease. In order to evaluate the performance of our method, the ten-fold cross validation and de novo test are implemented in experiment. We compare our method with other two the-stateof-the-art methods in term of AUC. The experimental results demonstrate that our method outperform other methods in prediction performance. The case study shows that our method can effectively identify potential miRNAdisease associations.

\section{RESULTS}

\section{Evaluation}

In this paper, ten-fold cross-validation and de novo test are utilized to evaluate the performance of our method. In the ten-fold cross-validation, all known miRNA-disease interactions are randomly divided into ten folds. For each cross validation round, one subset is treated as test samples and the rest nine subsets are regarded as training samples. After completing the test, predicted scores are generated. Then, we rank test samples and unknown miRNA-disease interactions. The corresponding predicted result of test samples is considered as true positive (TP) when the predicted relevance score is greater than the threshold. Otherwise, considered as false negative (FN). Similar, for the unknown miRNA-disease interactions, the corresponding predicted result consider as false positive (FP) when the predicted relevance score is greater than the threshold. Otherwise, considered as true negative (TN). Then, FPR (false positive rates) and TPR (true positive rates) are calculated as follow:

$$
\begin{aligned}
& T P R=\frac{T P}{T P+F N}, \\
& F P R=\frac{T N}{T N+F P} .
\end{aligned}
$$

Finally, the receiver operating characteristic (ROC) curve is drew and the area under the ROC curve (AUC) is 
calculated to evaluate the performance of method. The higher AUC value is, the better performance is.

\section{Comparison with different predicted methods using ten-fold cross validation}

We compare MDVSI with two the-state-of-the-art algorithms (LRLSLDA [15] and BRWH [18]). The result is shown in Figure 1. It can be observed from Figure 1 that MDVSI, LRLSLDA and BRWH achieve AUC of 0.9346, 0.9069 and 0.8393 , respectively. It demonstrates that MDVSI outperforms other two methods in the prediction performance.

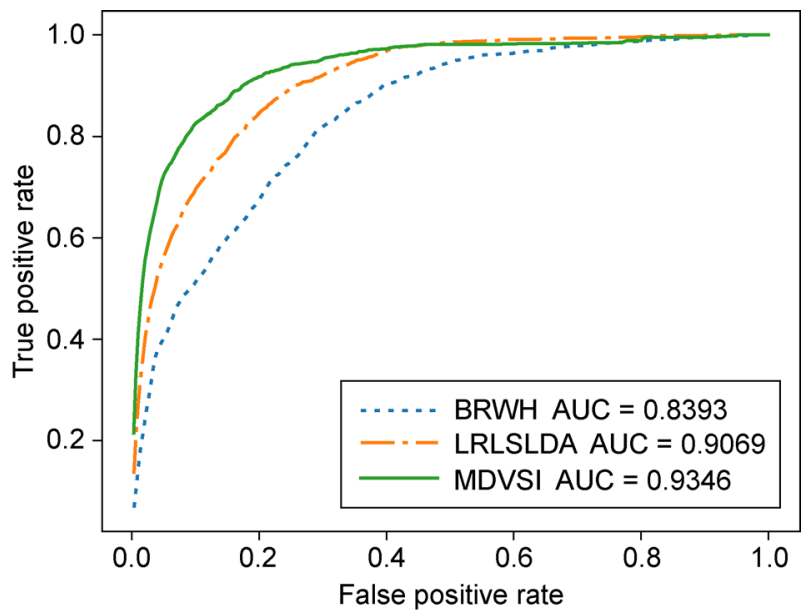

Figure 1. Comparison between MDVSI, LRLSLDA and BRWH by using ten-fold cross validation.

\section{Comparison with different predicted methods using de novo test}

To evaluate the ability of MDVSI in predicting interactions between miRNAs and new diseases, we conduct the de novo test here. In the de novo test, all the known miRNA-disease associations of one disease are removed. Then, the interactions are predicted by prediction method. It can be found in Figure 2 that MDVSI achieves AUC of 0.7468 which is 0.003 and 0.01 higher than LRLSLDA and BRWH, respectively. It demonstrates that MDVSI is an effective method for predicting interactions between new disease and miRNAs.

\section{The effect of parameter $\beta$}

We also test the effect of parameter $\beta$, which is used to balance the miRNA topological similarity and miRNA functional similarity in miRNA similarity integration process. The result is shown in the Table 1. It is easy to

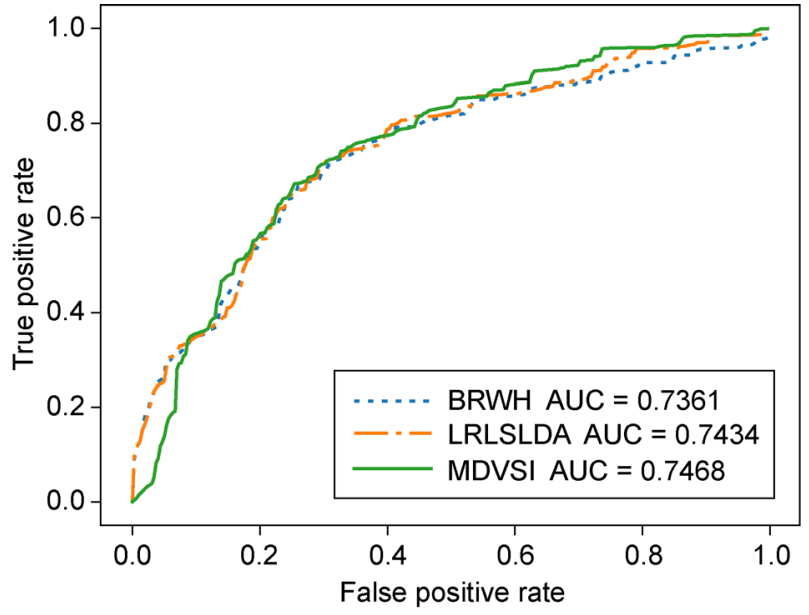

Figure 2. Comparison between MDVSI, LRLSLDA and BRWH by using de novo test.

Table 1 Effect of parameters on performance of MDVSI

\begin{tabular}{ll}
\hline Parameter & AUC \\
\hline 0 & 0.9311 \\
0.1 & 0.9340 \\
0.2 & 0.9312 \\
0.3 & 0.9333 \\
0.4 & 0.9331 \\
0.5 & 0.9346 \\
0.6 & 0.9332 \\
0.7 & 0.9341 \\
0.8 & 0.9332 \\
0.9 & 0.9332 \\
\hline
\end{tabular}

find that the MDVSI achieves the best performance in experimental result when $\beta=0.5$.

\section{The effect of parameter $k$}

In order to test the effect of parameter $k$, which is used to selecte the predicted interactions between miRNAs and new disease. The result is shown in the Table 2. It can be concluded that our method achieves the best performance when $k$ ranges from 17 to 19 .

\section{Case studies}

In order to validate the reliability of miRNAs predicted by our MDVSI, we select two important human cancers (lung cancer and breast cancer) as two case studies. We choose the top 50 predicted miRNAs of breast cancer and lung cancer, respectively. Then, we confirm these miRNAs by consulting databases and recent literatures. 
Table 2 Effect of parameters $k$ on performance

\begin{tabular}{ll}
\hline$k$ & AUC \\
\hline 10 & 0.7195 \\
11 & 0.7312 \\
12 & 0.7331 \\
13 & 0.7376 \\
14 & 0.7373 \\
15 & 0.7369 \\
16 & 0.7451 \\
17 & 0.7467 \\
18 & 0.7467 \\
19 & 0.7467 \\
20 & 0.7466 \\
\hline
\end{tabular}

Lung cancer is a kind of high death rate cancer for human. It has been proved that miRNAs have important roles in lung cancer. For example, the expression of miRNAs (has-miR-205, has-miR-19a, has-miR-19b, hasmiR-30b and has-miR-20a) decreased strikingly after lung SCC surgery [22]. The top-50 predicted miRNAs related with lung cancer are shown in Table 3 . It can be approved that 46 out of top 50 on the list are verified in recent literatures. We also discover some interesting miRNAs, such as hsa-mir-127, hsa-mir-9 and hsa-mir146b. It demonstrates that our algorithm can not only effectively predict known relationships, but also can predict the potential relationships between diseases and miRNAs.

Breast cancer accounts for three percent of human malignancies and the mortality rate continues to increase over the past years. It is the most common malignancies in women all over the world. Accumulating researches have shown that miRNAs play key roles in breast cancer. For example, some studies have found that the higher expression of mature miRNAs (hsa-miR-103, hsa-miR21-5p, hsa-miR-141, hsa-miR-25, hsa-miR-30b, hsamiR-30c, and hsa-let-7i) contribute to breast cancer survival [23]. As shown in Table 4, 43 out of top 50 are verified by consulting published literatures. The results demonstrate that MDVSI is a useful tool for identifying the potential disease-associated miRNAs.

\section{CONCLUSIONS}

Increasing experimental studies have indicated that

Table 3 The top 50 potential lung cancer related miRNAs predicted by MDVSI

\begin{tabular}{|c|c|c|c|c|c|}
\hline Rank & MiRNA & Evidence & Rank & MiRNA & Evidence \\
\hline 1 & hsa-let-7d & PMID: 30675288 & 26 & hsa-mir-218 & PMID: 28830450 \\
\hline 2 & hsa-let-7f & PMID: 24130905 & 27 & hsa-mir-126 & PMID: 22009180 \\
\hline 3 & hsa-let-7e & PMID: 24130905 & 28 & hsa-mir-30d & PMID: 25342220 \\
\hline 4 & hsa-let-7c & PMID: 23464461 & 29 & hsa-mir-34c & PMID: 30142158 \\
\hline 5 & hsa-let-7a & PMID: 25214829 & 30 & hsa-mir-9 & Unknown \\
\hline 6 & hsa-let-7b & PMID: 29849986 & 31 & hsa-mir-29a & PMID: 26676674 \\
\hline 7 & hsa-let-7i & Unknown & 32 & hsa-mir-30b & PMID: 25344866 \\
\hline 8 & hsa-mir-19b & PMID:24130905 & 33 & hsa-mir-214 & PMID: 28396596 \\
\hline 9 & hsa-mir-145 & PMID: 28927412 & 34 & hsa-mir-223 & PMID: 29163821 \\
\hline 10 & hsa-mir-132 & PMID: 28321148 & 35 & hsa-mir-128b & PMID: 28514100 \\
\hline 11 & hsa-mir-17 & PMID:27735039 & 36 & hsa-mir-192 & PMID: 21511813 \\
\hline 12 & hsa-mir-18a & PMID: 28790336 & 37 & hsa-mir-196a & PMID: 18521189 \\
\hline 13 & hsa-mir-199a & PMID: 30233883 & 38 & hsa-mir-92a & PMID: 26893365 \\
\hline 14 & hsa-mir-191 & PMID: 20169152 & 39 & hsa-mir-146b & Unknown \\
\hline 15 & hsa-mir-30c & PMID: 25344866 & 40 & hsa-mir-155 & PMID: 29065541 \\
\hline 16 & hsa-mir-34a & PMID: 23805317 & 41 & hsa-mir-32 & PMID: 25755781 \\
\hline 17 & hsa-mir-125a & PMID: 20569443 & 42 & hsa-mir-24 & PMID: 29850625 \\
\hline 18 & hsa-let-7g & PMID: 26316738 & 43 & hsa-mir-200b & PMID: 25279705 \\
\hline 19 & hsa-mir-29b & PMID: 24130905 & 44 & hsa-mir-106a & PMID: 24130905 \\
\hline 20 & hsa-mir-34b & PMID: 28869603 & 45 & hsa-mir-106b & PMID: 27477696 \\
\hline 21 & hsa-mir-19a & PMID: 22303398 & 46 & hsa-mir-429 & PMID: 24523873 \\
\hline 22 & hsa-mir-101 & PMID: 25210796 & 47 & hsa-mir-219 & PMID: 28790336 \\
\hline 23 & hsa-mir-20a & PMID: 26672767 & 48 & hsa-mir-25 & PMID: 29568911 \\
\hline 24 & hsa-mir-30e & PMID: 28178679 & 49 & hsa-mir-127 & Unknown \\
\hline 25 & hsa-mir-205 & PMID: 22303398 & 50 & hsa-mir-210 & PMID: 27557519 \\
\hline
\end{tabular}


Table 4 The top 50 potential breast cancer related miRNAs predicted by MDVSI

\begin{tabular}{|c|c|c|c|c|c|}
\hline Rank & MiRNA & Evidence & Rank & MiRNA & Evidence \\
\hline 1 & hsa-mir-429 & PMID: 30594253 & 26 & hsa-mir-127 & PMID: 27983524 \\
\hline 2 & hsa-mir-25 & PMID: 27959953 & 27 & hsa-mir-9 & PMID: 30502282 \\
\hline 3 & hsa-mir-218 & PMID: 29378184 & 28 & hsa-mir-18a & PMID: 30817902 \\
\hline 4 & hsa-mir-367 & PMID: 21810988 & 29 & hsa-mir-219 & PMID: 23813567 \\
\hline 5 & hsa-mir-93 & PMID: 28765915 & 30 & hsa-mir-339 & PMID: 30683807 \\
\hline 6 & hsa-let-7d & Unknown & 31 & hsa-mir-30c & PMID: 25120384 \\
\hline 7 & hsa-mir-320 & PMID:29538612 & 32 & hsa-mir-153 & PMID: 28101798 \\
\hline 8 & hsa-let-7f & PMID: 18812439 & 33 & hsa-mir-194 & Unknown \\
\hline 9 & hsa-let-7a & PMID:29963109 & 34 & hsa-mir-17 & PMID: 28875846 \\
\hline 10 & hsa-mir-302c & Unknown & 35 & hsa-mir-296 & PMID: 24527800 \\
\hline 11 & hsa-mir-215 & PMID:30194145 & 36 & hsa-mir-145 & PMID: 30704524 \\
\hline 12 & hsa-mir-199a & PMID: 30001527 & 37 & hsa-let-7e & Unknown \\
\hline 13 & hsa-mir-302b & PMID: 26842910 & 38 & hsa-mir-214 & PMID: 27422604 \\
\hline 14 & hsa-mir-200a & PMID: 30786836 & 39 & hsa-mir-19a & Unknown \\
\hline 15 & hsa-mir-19b & PMID: 30343695 & 40 & hsa-mir-34a & Unknown \\
\hline 16 & hsa-mir-302a & PMID: 29435003 & 41 & hsa-let-7b & PMID: 29849944 \\
\hline 17 & hsa-mir-106b & PMID: 30348127 & 42 & hsa-mir-10b & PMID: 30250531 \\
\hline 18 & hsa-mir-302d & PMID: 26644266 & 43 & hsa-mir-34b & PMID: 27557899 \\
\hline 19 & hsa-mir-30d & PMID: 29923255 & 44 & hsa-mir-103 & PMID: 28320108 \\
\hline 20 & hsa-mir-200b & PMID: 30847025 & 45 & hsa-mir-141 & PMID: 28440475 \\
\hline 21 & hsa-mir-125a & PMID: 30670152 & 46 & hsa-mir-20a & PMID: 30092355 \\
\hline 22 & hsa-mir-30b & PMID: 30154547 & 47 & hsa-mir-383 & Unknown \\
\hline 23 & hsa-mir-151 & PMID: 27930738 & 48 & hsa-mir-338 & PMID: 26252944 \\
\hline 24 & hsa-mir-488 & PMID: 27609814 & 49 & hsa-let-7i & PMID: 24699530 \\
\hline 25 & hsa-mir-135b & PMID: 30665445 & 50 & hsa-let-7c & PMID: 24866763 \\
\hline
\end{tabular}

miRNAs play an important role in various biological processes $[24,25]$. Therefore, it is important to effectively identify the relationship between miRNA and disease $[26,27]$. While existing experimental methods for miRNA-disease interaction identification are generally time-consuming and unable to apply to large scale datasets. Therefore, it is demanded to develop computational method for predicting miRNA-disease associations. In the paper, we propose a novel method (MDVSI) to infer relationships between miRNAs and diseases. Firstly, we utilize disease-gene interaction downloaded from HumanNet to calculate disease-disease functional similarity. Then, the miRNA topological similarity is calculated based on miRNA-disease network topological feature. Further, the miRNA functional similarity and miRNA topological similarity are integrated to improve the accuracy of prediction by using recommendation method. In addition, in order to overcome the limitation of recommendation method in predicting interaction of new disease, we propose a new method to predict interactions between miRNAs and new disease based on disease similarity. To evaluate the prediction performance, we compare our method with other two algorithms via using ten-fold cross-validation and de novo test. Experimental results show that our model has superior performance than other compared methods for predicting miRNAdisease associations.

\section{MATERIALS AND METHODS}

\section{Datasets}

The miRNA-disease association is downloaded from HMDD [28]. In the final, we select 271 miRNAs, 137 diseases and 1,395 miRNA-disease associations as gold dataset. The adjacency matrix $A$ is constructed to represent the relationships between miRNAs and diseases, where $A(i, j)=1$ if miRNA $i$ is associated with disease $j$, otherwise $A(i, j)=0$.

We extract disease functional similarity from HumanNet dataset [29], which contains 16,243 genes and 476,399 interactions. The assigned score for paired genes in HumanNet represent the gene functional similarity.

The miRNA functional similarity is downloaded from misim [30]. Then, the miRNA-miRNA functional 
similarity network is described as $F M$ matrix based on the calculated score. The element of matrix $F M(i, j)$ denotes the similarity score between miRNAs $i$ and $j$.

\section{Methods}

We propose a computational framework, MDVSI, for miRNA-disease association identification. In the first step, we calculate disease functional similarity based on disease-gene interaction. Then, the miRNA functional similarity and miRNA topological similarity are calculated based on HumanNet and miRNA-disease network topological feature, respectively. Further, the miRNA functional similarity and miRNA topological similarity are integrated to enhance the reliability of miRNA similarity. Finally, the potential miRNA-disease associations are predicted by using recommendation method. In addition, to overcome the limitation of recommendation method for predicting interaction of new disease, we predict associations between new disease and miRNAs based on disease functional similarity. The flowchart of MDVSI is shown in Figure 3.

The disease-disease similarity

It has been proved that human disease has close relationships with gene. Therefore, based on the disease-gene association, we calculate the functional similarity of diseases. For the two genes $g_{i}$ and $g_{j}$, the similarity between $g_{i}$ and $g_{j}$ is defined as follow:

$$
\begin{aligned}
& e w\left(g_{i}, g_{j}\right) \\
& = \begin{cases}1 & \text { if } g_{i}=g_{j} \\
c c\left(g_{i}, g_{j}\right) & \text { if } g_{i} \neq g_{j}, \quad g_{i} \text { and } g_{j} \text { connected } \\
0 & \text { if } g_{i} \neq g_{j}, \quad g_{i} \text { and } g_{j} \text { not connected }\end{cases}
\end{aligned}
$$

where $e w\left(g_{i}, g_{j}\right)$ denotes the similarity between $g_{i}$ and $g_{j}$. $c c\left(g_{i}, g_{j}\right)$ denotes $\log$ likelihood score between $g_{i}$ and $g_{j}$ which is calculated in HumanNet.

Given two gene sets $G_{1}=\left\{g_{11}, g_{12}, \ldots, g_{1 m}\right\}$ and $G_{2}$ $=\left\{g_{21}, g_{22}, \ldots, g_{2 n}\right\}$ associated with disease $d_{1}$ and disease $d_{2}$, respectively. Then, the score between gene $g_{2 j}$ and disease $d_{1}$ is defined as follow:

$$
\begin{aligned}
& G F_{d_{1}}\left(g_{2 j}\right)=\max \sum_{i=1}^{m}\left(e w\left(g_{1 i}, g_{2 j}\right)\right), g_{1 i} \in G_{1}, \\
& G F_{d 2}\left(g_{1 i}\right)=\max \sum_{j=1}^{n}\left(e w\left(g_{1 i}, g_{2 j}\right)\right), g_{2 j} \in G_{2},
\end{aligned}
$$

In the same way, the $G F_{d 2}\left(g_{1 i}\right)$ can also be calculated.

Finally, the similarity of diseases $d_{1}$ and $d_{2}$ is defined as follow:

$$
S D\left(d_{1}, d_{2}\right)=\frac{\sum_{j=1}^{n} G F_{d_{1}}\left(g_{2 j}\right)+\sum_{i=1}^{m} G F_{d_{2}}\left(g_{1 i}\right)}{m+n} .
$$

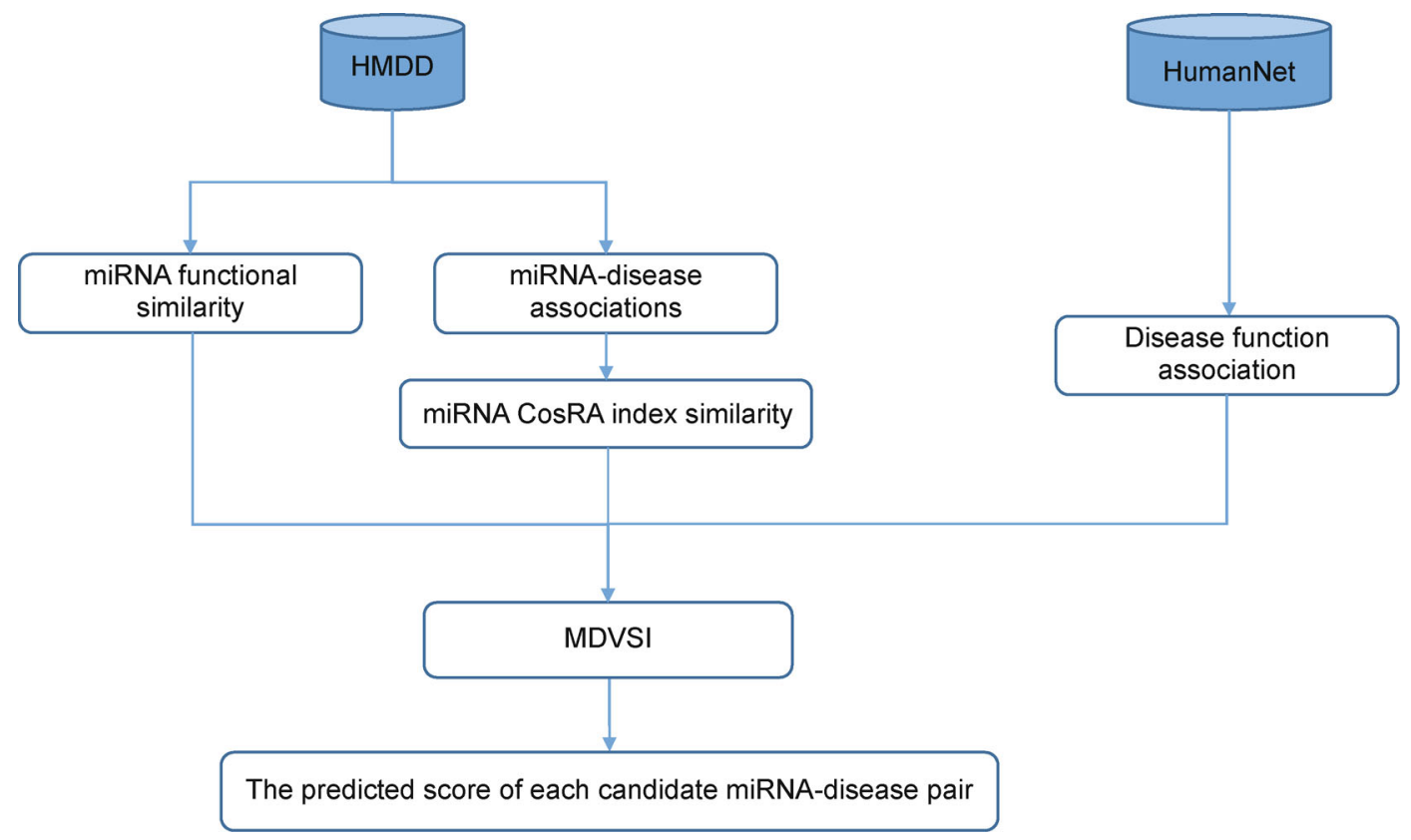

Figure 3. The flow chart for miRNA-disease interactions identification by using MDVSI. 
The miRNA-miRNA topological similarity

Considering that miRNA-disease interaction may reflect topological feature, miRNA topological similarity is calculated by using CosRA index [31]. The miRNA topological similarity between miRNA $i$ and $j$ is defined as follows:

$$
T M^{\operatorname{Cos} R A}(i, j)=\frac{1}{\sqrt{k_{i} k_{j}}} \sum_{x=1}^{p} \frac{a_{i x} a_{j x}}{k_{x}},
$$

where $k_{i}$ and $k_{j}$ denote the degree of miRNA $i$ and $j$, respectively. $k_{x}$ denotes the degree of disease $x . a_{i x}$ represents the interaction between miRNA $i$ and disease $x$ $\left(a_{i x}=1\right.$ if miRNA $i$ is related with disease $x$, otherwise $\left.a_{i x}=0\right) . a_{j x}$ denotes the interaction between miRNA $j$ and disease $x\left(a_{j x}=1\right.$ if miRNA $j$ is related with disease $x$, otherwise $a_{j x}=0$ ). $p$ is the sum number of diseases.

Integrate miRNA similarities

In order to improve the reliability of miRNA similarity, we integrate miRNA functional similarity and miRNA topological similarity as follows:

$$
S M=\beta * T M^{\operatorname{Cos} R A}+(1-\beta) * F M,
$$

where $F M$ denotes the functional similarity of miRNA. $\beta$ is the parameter to balance two similarities in fusion process.

Predict potential miRNA-disease association

We predict potential miRNA-disease association based on recommendation method [32], which is used to calculate the miRNA-target prediction. According to the known relationships between miRNA and disease, the predicted score of potential relationships between miRNA $i$ and disease $j$ is calculated as follows:

$$
\operatorname{Score}(i, j)=S M(i, *) \times A(*, j),
$$

where $S M$ denotes the miRNA similarity matrix. $A$ denotes the miRNA-disease association matrix. The higher score is, the closer relationship between miRNA $i$ and disease $j$ is.

Predict interaction between miRNA and new disease

It is well known that the recommendation method is unable to predict new entity (i.e., cold start problem). In order to overcome this limitation, we propose a new strategy for predicting potential interactions between miRNAs and new disease $i$. For new disease $i$, the top $k$ most similarity diseases are selected based on disease functional similarity. And then it is considered to be associated with miRNA $j$ if over two similar diseases have relationship with the miRNA $j$. We use these associations as known knowledge and predict the potential relationships between new disease $i$ and miRNAs by using recommendation method.

\section{ACKNOWLEDGEMENTS}

The work reported in this paper was partially supported by the National Natural Science Foundation of China (Nos. 61702122, 61751314 and 31560317), the Natural Science Foundation of Guangxi (Nos. 2017GXNSFDA198033 and 2018GXNSFBA281193), the Key Research and Development Plan of Guangxi (No. AB17195055), the Bossco Project of Guangxi University (No. 20190240), the Hunan Provincial Science and Technology Program (No. 2018WK4001) and 111 Project (No. B18059).

\section{COMPLIANCE WITH ETHICS GUIDELINES}

The authors Qingfeng Chen, Zhao Zhe, Wei Lan, Ruchang Zhang, Zhiqiang Wang, Cheng Luo and Yi-Ping Pheobe Chen declare that they have no conflict of interests.

This article does not contain any studies with human or animal subjects performed by any of the authors.

\section{REFERENCES}

1. Ambros, V. (2004) The functions of animal microRNAs. Nature, 431, 350-355

2. Akhtar, M. M., Micolucci, L., Islam, M. S., Olivieri, F. and Procopio, A. D. (2016) Bioinformatic tools for microRNA dissection. Nucleic Acids Res., 44, 24-44

3. Iorio, M. V., Ferracin, M., Liu, C. G., Veronese, A., Spizzo, R., Sabbioni, S., Magri, E., Pedriali, M., Fabbri, M., Campiglio, M., et al. (2005) MicroRNA gene expression deregulation in human breast cancer. Cancer Res., 65, 7065-7070

4. Ashmawy, A. M., Elgeshy, K. M., Abdel Salam, E. T., Ghareeb, M., Kobaisi, M. H., Amin, H. A. A., Sharawy, S. K. and Abdel Wahab, A. H. A. (2017) Crosstalk between liver-related microRNAs and Wnt/ $\beta$-catenin pathway in hepatocellular carcinoma patients. Arab J. Gastroenterol., 18, 144-150

5. Kim, Y. K., Yu, J., Han, T. S., Park, S. Y., Namkoong, B., Kim, D. H., Hur, K., Yoo, M. W., Lee, H. J., Yang, H. K., et al. (2009) Functional links between clustered microRNAs: suppression of cell-cycle inhibitors by microRNA clusters in gastric cancer. Nucleic Acids Res., 37, 1672-1681

6. Moss, E. G., Lee, R. C. and Ambros, V. (1997) The cold shock domain protein LIN-28 controls developmental timing in $C$. elegans and is regulated by the lin-4 RNA. Cell, 88, 637-646

7. Lan, W., Chen, Q., Li, T., Yuan, C., Mann, S. and Chen, B. (2014) Identification of important positions within miRNAs by integrating sequential and structural features. Curr. Protein Pept. Sci., 15, 591597

8. Chen, Q., Lan, W., Wang, J. X. (2013) Mining featured patterns of miRNA interaction based on sequence and structure similarity. 
IEEE/ACM Trans. Comput. Biol. Bioinform., 10, 415-422

9. Luo, H., Lan, W., Chen, Q., Wang, Z., Liu, Z., Yue, X. and Zhu, L. (2018) Inferring microRNA-environmental factor interactions based on multiple biological information fusion. Molecules, 23, 2439

10. Lan, W., Wang, J. X., Li, M., Liu, J. and Pan, Y. (2015) Predicting microRNA-disease associations by integrating multiple biological information. In: EEE International Conference on Bioinformatics and Biomedicine (BIBM), pp.183-188

11. Peng, W., Lan, W., Zhong, J., Wang, J. X. and Pan, Y. (2017) A novel method of predicting microRNA-disease associations based on microRNA, disease, gene and environment factor networks. Methods, 124, 69-77

12. Jiang, Q., Hao, Y., Wang, G., Juan, L., Zhang, T., Teng, M., Liu, Y. and Wang, Y. (2010) Prioritization of disease microRNAs through a human phenome-microRNAome network. BMC Syst. Biol., 4, S2

13. Xuan, P., Han, K., Guo, M., Guo, Y., Li, J., Ding, J., Liu, Y., Dai, Q., Li, J., Teng, Z., et al. (2013) Prediction of microRNAs associated with human diseases based on weighted $k$ most similar neighbors. PLoS One, 8, e70204

14. Mørk, S., Pletscher-Frankild, S., Palleja Caro, A., Gorodkin, J. and Jensen, L. J. (2014) Protein-driven inference of miRNA-disease associations. Bioinformatics, 30, 392-397

15. Chen, X. and Yan, G. Y. (2014) Semi-supervised learning for potential human microRNA-disease associations inference. Sci. Rep., 4, 5501

16. Peng, W., Lan, W., Yu, Z., Wang, J., Pan, Y. (2016) A Framework for integrating multiple biological networks to predict microRNAdisease associations. IEEE Trans. Nanobio., 16, 100-107

17. Yan, C., Wang, J., Ni, P., Lan, W., Wu, F. X., Pan, Y. (2019) DNRLMF-MDA: predicting microRNA-disease associations based on similarities of microRNAs and diseases. IEEE/ACM Trans. Comput. Biol. Bioinform., 16, 233-243

18. Luo, J. and Xiao, Q. (2017) A novel approach for predicting microRNA-disease associations by unbalanced bi-random walk on heterogeneous network. J. Biomed. Inform., 66, 194-203

19. Lan, W., Wang, J. X., Li, M., Liu, J., Wu, F.X., Pan, Y. (2018) Predicting microRNA-disease associations based on improved microRNA and disease similarities. IEEE/ACM Trans. Comput. Biol. Bioinform., 15, 1774-1782

20. Liu, Y., Zeng, X., He, Z., Zou, Q. (2016) Inferring microRNAdisease associations by random walk on a heterogeneous network with multiple data sources. IEEE/ACM Trans. Comput. Biol. Bioinform., 14, 905-915

21. Zou, Q., Li, J., Hong, Q., Lin, Z., Wu, Y., Shi, H. and Ju, Y. (2015) Prediction of microRNA-disease associations based on social network analysis methods. BioMed Res. Int., 2015, 810514

22. Aushev, V. N., Zborovskaya, I. B., Laktionov, K. K., Girard, N., Cros, M. P., Herceg, Z. and Krutovskikh, V. (2013) Comparisons of microRNA patterns in plasma before and after tumor removal reveal new biomarkers of lung squamous cell carcinoma. PLoS One, 8, e78649

23. Chang, J. T., Wang, F., Chapin, W. and Huang, R. S. (2016) Identification of microRNAs as breast cancer prognosis markers through the Cancer Genome Atlas. PLoS One, 11, e0168284

24. Köhler, S., Bauer, S., Horn, D. and Robinson, P. N. (2008) Walking the interactome for prioritization of candidate disease genes. Am. J. Hum. Genet., 82, 949-958

25. Lan, W., Li, M., Zhao, K., Liu, J., Wu, F. X., Pan, Y. and Wang, J. (2017) LDAP: a web server for lncRNA-disease association prediction. Bioinformatics, 33, 458-460

26. Lan, W., Huang, L., Lai, D., and Chen, Q. (2018) Identifying Interactions Between Long Noncoding RNAs and Diseases Based on Computational Methods. In: Computational Systems Biology, pp.205-221, Springer Nature

27. Lan, W., Wang, J. X., Li, M., Peng, W. and Wu, F. (2015) Computational approaches for prioritizing candidate disease genes based on PPI networks. Tsinghua Sci. Technol., 20, 500-512

28. Li, Y., Qiu, C., Tu, J., Geng, B., Yang, J., Jiang, T. and Cui, Q. (2014) HMDD v2.0: a database for experimentally supported human microRNA and disease associations. Nucleic Acids Res., 42, D1070-D1074

29. Hwang, S., Kim, C. Y., Yang, S., Kim, E., Hart, T., Marcotte, E. M. and Lee, I. (2019) HumanNet v2: human gene networks for disease research. Nucleic Acids Res., 47, D573-D580

30. Wang, D., Wang, J., Lu, M., Song, F. and Cui, Q. (2010) Inferring the human microRNA functional similarity and functional network based on microRNA-associated diseases. Bioinformatics, 26, 1644-1650

31. Chen, L. J., Zhang, Z. K., Liu, J. H., Gao, J. and Zhou, T. (2017) A vertex similarity index for better personalized recommendation. Physica A, 466, 607-615

32. Jiang, H., Wang, J., Li, M., Lan, W., Wu, F., Pan, Y. (2018) miRTRS: a recommendation algorithm for predicting miRNA targets. IEEE/ACM Trans. Comput. Biol. Bioinform., 1 